\title{
Interpretation of the "Catastrophe" of Quasars on the Basis of New Decisions of the Problem of the Reverse Compton-Effect
}

\author{
Nikolay I. Perov ${ }^{1}$, Artem Eltsov ${ }^{2}$ \\ 1. State Autonomous Organization of Culnure and Education named after V.V. Tereshkova, Yaroslavl, 150000, Russian Federation \\ 2. State Pedagogical University named after K.D. Ushinskii, Yaroslavl,150000, Russian Federation
}

\begin{abstract}
In a model based on a search with increased accuracy of calculations-four times-of all the roots of the equations for the velocities of electrons in the inverse Compton effect, it is established that for new values of the roots of the equations (corresponding to the laws of conservation of momentum and energy), the recoil electrons remain ultra-relativistic, according to the authors, allows to eliminate the reverse Compton catastrophe of quasars. The maximum value of the scattering angle of the formed particles was found to explain the thin quasar jets.
\end{abstract}

Keywords: Quasar 3C273, critical temperature, inverse Compton effect, ultra relativistic electrons, limiting angle of expansion.

\section{Introduction}

Observations established the temperature $\mathrm{T}_{\mathrm{H}}=4$. $10^{13} \mathrm{~K}$ of the quasar $3 \mathrm{C} 273$ [1], which is an order of magnitude higher than the theoretically possible value of the effective quasar temperature $\left(\mathrm{T}_{\mathrm{m}}=5 \cdot 10^{11} \mathrm{~K}\right)$.

The existence of a critical value of the temperature $\mathrm{Tm}$ is associated with the inverse Compton catastrophe: if the electron energy exceeds a certain limit, then they begin to transmit avalanche energy to the photons and cool down. In this case, jets (plasma) from high-energy particles near quasars should not be observed. But the quasar $3 \mathrm{C} 273$ violates this restriction [2]. Let us show that the recoil electrons can also be ultra-relativistic, whose velocity $\mathrm{V}_{2}$ differs little from the speed of light $(\mathrm{c}=299792458 \mathrm{~m} / \mathrm{s})$, and the new photons correspond to the temperature $T_{n}$.

\section{Fundamental Equations}

Let us assume that as a result of a "head-on" collision of a relativistic electron of mass $m$ having a

Corresponding author: Artem Eltsov, assistant professor, research fields: astronomy, astrophysics. velocity $\mathrm{V}_{1}$ and a relic photon characterized by a wavelength $\lambda_{1}=1.9 \mathrm{~mm}$ and a frequency $v_{1}=\mathrm{c} / \lambda_{1}$, a recoil electron moving at a velocity of $\mathrm{V}_{2}$ and a photon $\lambda_{2}$, frequency $v_{2}$ ), corresponding to the emission of an absolutely black body with a temperature $\mathrm{Tn}$. We denote the angle between the momenta of the "new" photon and the recoil electron by $\theta$. Let us find the electron velocities $V_{1}$ and $V_{2}$ [3], [4], [5].

Based on the law of conservation of energy, we have

$$
\frac{\mathrm{mc}^{2}}{\sqrt{1-\frac{\mathrm{V}_{1}^{2}}{\mathrm{c}^{2}}}}+\mathrm{h} v_{1}=\frac{\mathrm{mc}^{2}}{\sqrt{1-\frac{\mathrm{V}_{2}^{2}}{\mathrm{c}^{2}}}}+\mathrm{h} v_{2}
$$

The law of conservation of momentum gives

$$
\left(\frac{\mathrm{mV}_{1}}{\sqrt{1-\frac{\mathrm{V}_{1}^{2}}{\mathrm{c}^{2}}}}-\frac{\mathrm{h} \mathrm{v}_{1}}{\mathrm{c}}\right)^{2}=
$$

$$
\frac{\mathrm{m}^{2} \mathrm{~V}_{2}^{2}}{1-\frac{\mathrm{V}_{2}^{2}}{\mathrm{c}^{2}}}+\left(\frac{\mathrm{h} v_{2}}{\mathrm{c}}\right)^{2}+2 \frac{\mathrm{mV} \mathrm{V}_{2}}{\sqrt{1-\frac{\mathrm{V}_{2}^{2}}{\mathrm{c}^{2}}}} \frac{\mathrm{h} v_{2}}{\mathrm{c}} \cos \theta
$$


Here $\mathrm{h}$ is the Planck constant. To change from the temperature of the $T_{H}$ to the corresponding wavelength $\lambda$ (and the frequency $v$ ), we use the Wien law (for a Wien constant, $b=0.002897 \mathrm{~m} \cdot \mathrm{K}$ ).

\section{Results}

The particular solutions of equations (1) and (2), using computer algebra ("MAPLE-15") and 48 significant digits, are as follows:

a) at $\theta=0$

$V_{1}=0.99999999999992475405562886 \cdot \mathrm{c}$,

$V_{2}=0.99999999999992275921124240 \cdot \mathrm{c}$.

$V_{2}<V_{1}$,

$V_{1}-V_{2}=1.9948 \cdot 10^{-15} \cdot \mathrm{c}$

$E_{1}=1.317668819275908 \cdot 10^{12} \mathrm{eV}$,

$E_{2}=1.300542256694272 \cdot 10^{12} \mathrm{eV}$;

б) at $\theta_{\lim }=2.237 \cdot 10^{-5}$ degrees, or $\theta_{\lim }=0.0805 \mathrm{arc}$ seconds

$\mathrm{V}_{1}$

2.9979245799999999643777060251962165277687037 $34293 \cdot 10^{8}$,

$\mathrm{V}_{2}$

2.997924579999999643408794416590684523910398 $38245 \cdot 10^{8}$

$V_{l}=-2.997924580000228196739751715989910483$

$32087006383 \cdot 10^{8}$

$+5.96886604476345024967485772944946603117680$

$631289 \cdot 10^{-7} \sqrt{ }-1$

$\mathrm{V}_{2}$

2.997924580000228313820632467716121726850118 $86091 \cdot 10^{8}$

$-1.137813260468213809290060194987011748152$ $60843721 \cdot 10^{-20} \mathrm{~V}_{-}-1$.

$V_{l}=-2.997924580000228196739751715989910483$

$32087006383 \cdot 10^{8}$

$-5.96886604476345024967485772944946603117680$

$631289-10^{-7} \mathrm{~V}-1$,

$V_{2}=2.9979245800002283138206324677161217268$

$5011886091 \cdot 10^{8}$

$+1.13781326046821380929006019498701174815260$

$843721 \cdot 10^{-20} \sqrt{ }-1$.
$\mathrm{V}_{1}$ and $\mathrm{V}_{2}$ are measured in meters per second.

The actual values of the velocities are expressed in units of $\mathrm{c}$, then

$\mathrm{V}_{1}=0.999999999999999957472132 \cdot \mathrm{c}$,

$\mathrm{V}_{2}=0.999999999999999957445837 \cdot \mathrm{c}$.

$\mathrm{V}_{2}<\mathrm{V}_{1}$,

$\mathrm{V}_{1}-\mathrm{V}_{2}=2.6295 \cdot 10^{-20} \cdot \mathrm{c}$.

$\mathrm{E}_{1}=5.542567825934786 \cdot 10^{13} \mathrm{eV}$,

$\mathrm{E}_{2}=5.540855169676622 \cdot 10^{13} \mathrm{eV}$.

Here $\theta_{\lim }$ is the limiting (maximum) particle scattering angle (photon and recoil electron) at which the initial and final electron velocities have real, positive and ultra-relativistic values. $E_{1}$ and $E_{2}$ are the electron energies before the interaction with the photon and after the expansion of the photon and the electron.

c) $\theta=0.0806$ arc second

The actual values of the velocities $V_{1}$ and $V_{2}$ are absent. In this case, for both $\mathrm{V}_{1}$ and $\mathrm{V}_{2}$ only paired complex-conjugate roots are found.

$\mathrm{V}_{1}=2.9979245800000003093870875047460266351$

1119538071 . $10^{8}$

$-1.12051385274902127727133210325146185166171$

$210903 \quad \cdot 10^{-8} \quad V_{-} 1$,

$\mathrm{V}_{2}=2.997924580000000309549572738036883452980$

$06624625 \cdot 10^{8}$

$-1.117679160615765484111341328991528749460$

$01393449 \cdot 10^{-8} \sqrt{ }-1$

$\mathrm{V}_{1}$

$=-2.9979245800002287637486102380533306531848$

7276139 .

$10^{8}$

$+5.99113281763605213037461727680526739816412$

$649848 \cdot 10^{-7} \mathrm{~V}-1$,

$$
\mathrm{V}_{2}=2
$$

9979245800002288814122609371870362318703421

$1919 \cdot 10^{8}$

$-1.14205834259391486928760806009053218829304$

$632326 \cdot 10^{-20} \sqrt{ }-1$;

$\mathrm{V}_{1}=-2.99792458000022876374861023805333065318$

$487276139 \cdot 10^{8}$

$-5.99113281763605213037461727680526739816412$

$649848-10^{-7} \sqrt{ }-1$, 
$\mathrm{V}_{2}=2.997924580000228881412260937187036231870$ $34211919 \cdot 10^{8}$

$+1.14205834259391486928760806009053218829304$

$632326-10^{-20}$

$\sqrt{ }-1$

$\mathrm{V}_{1}=2.997924580000000309387087504746026635111$ 19538071 .

$+1.12051385274902127727133210325146185166171$

$210903 \cdot 10^{-8} \mathrm{~V}-1$,

$\mathrm{V}_{2}=2.9979245800000003095495727380368834$

$5298006624625 \cdot 10^{8}$

$+1.11767916061576548411134132899152874946001$ $393449^{-8} \mathrm{~V}-1$.

$\mathrm{V}_{1}$ and $\mathrm{V}_{2}$ are measured in meters per second.

In Fig. 1 and Fig. 2, taking into account Eqs. (1) and (2), graphs of the functions of the scattering angle $\theta$ of the photon and the recoil electron from the initial velocity of the electron $V_{1}$ (Fig. 1) and the scattering angle $\theta$ of the photon and the "recoil" electron from the velocity of the "recoil" electron $V_{2}$ (Fig. 2). Let's pay attention to small values of admissible angles of expansion of photons and electrons (0.08 ") and values of speeds $V_{1}$ and $V_{2}$ differing little from speed of light c.

\section{Conclusions}

Thus, in this model, the recoil electrons remain relativistic (ultra-relativistic $-\mathrm{V}_{1} \approx \mathrm{V}_{2} \approx \mathrm{c}$ ). It is possible that the above arguments will solve the problem of the inverse Compton catastrophe in the case of quasars (since the approximate equality $\mathrm{V}_{1} \approx$ $\mathrm{V}_{2} \approx \mathrm{c}$ has been proved), and also to clarify the physical nature of the thin jets existing near quasars (the angle $\theta$ is small, $\theta \approx 0.0805$ ").

Note. When $\theta=0$, Eq. (2) reduces to a linear equation with respect to the particle momenta, taking this circumstance into account, the general letter solution of Eqs. (1) and (2), found using computer algebra (MAPLE-15), looks like:

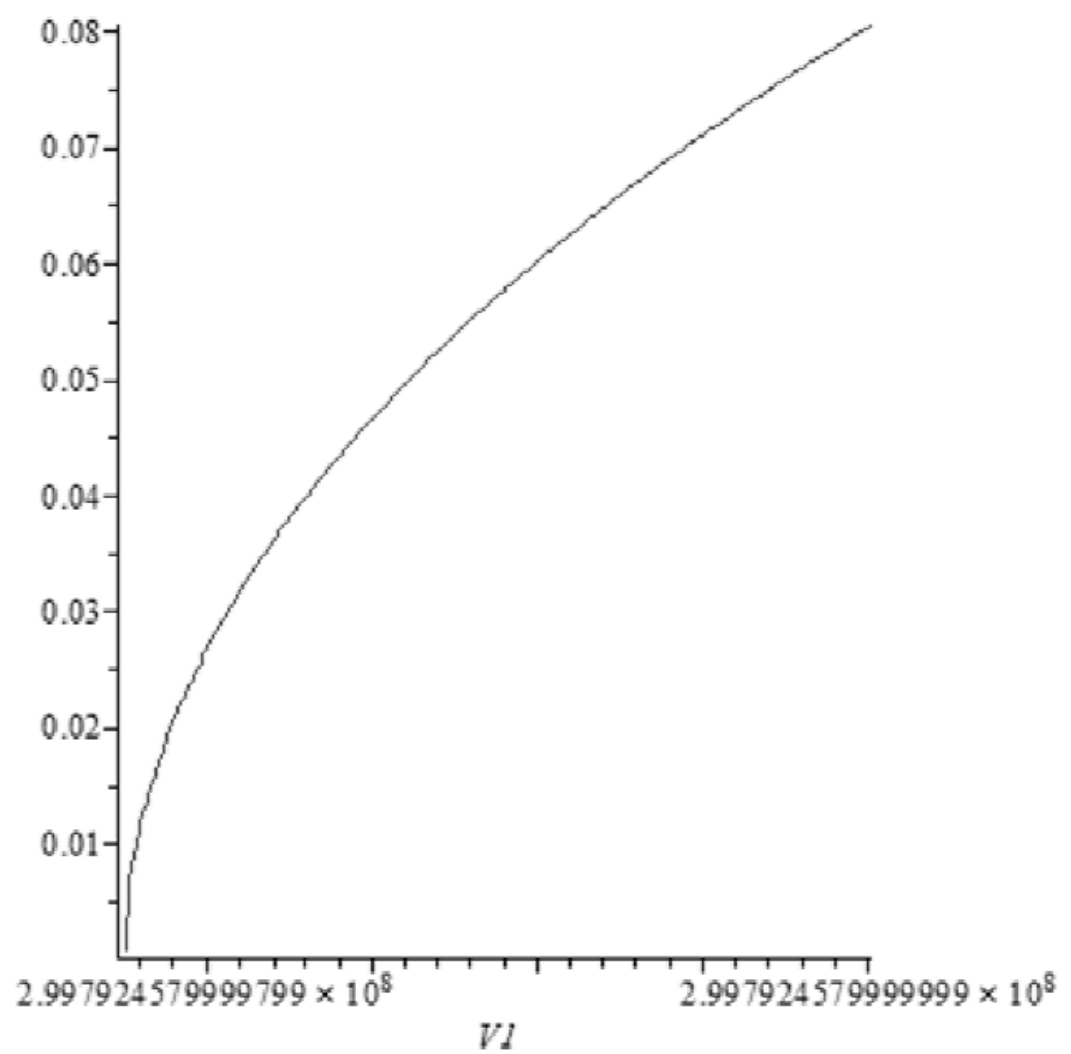

Fig. 1 Dependence of the angle $\theta$ of the photon and the "recoil" electron on the initial velocity of the electron $\left(V_{1}\right)$. The speed of an electron is measured in meters per second. The angle of dispersion $\theta$ is measured in angular seconds. 


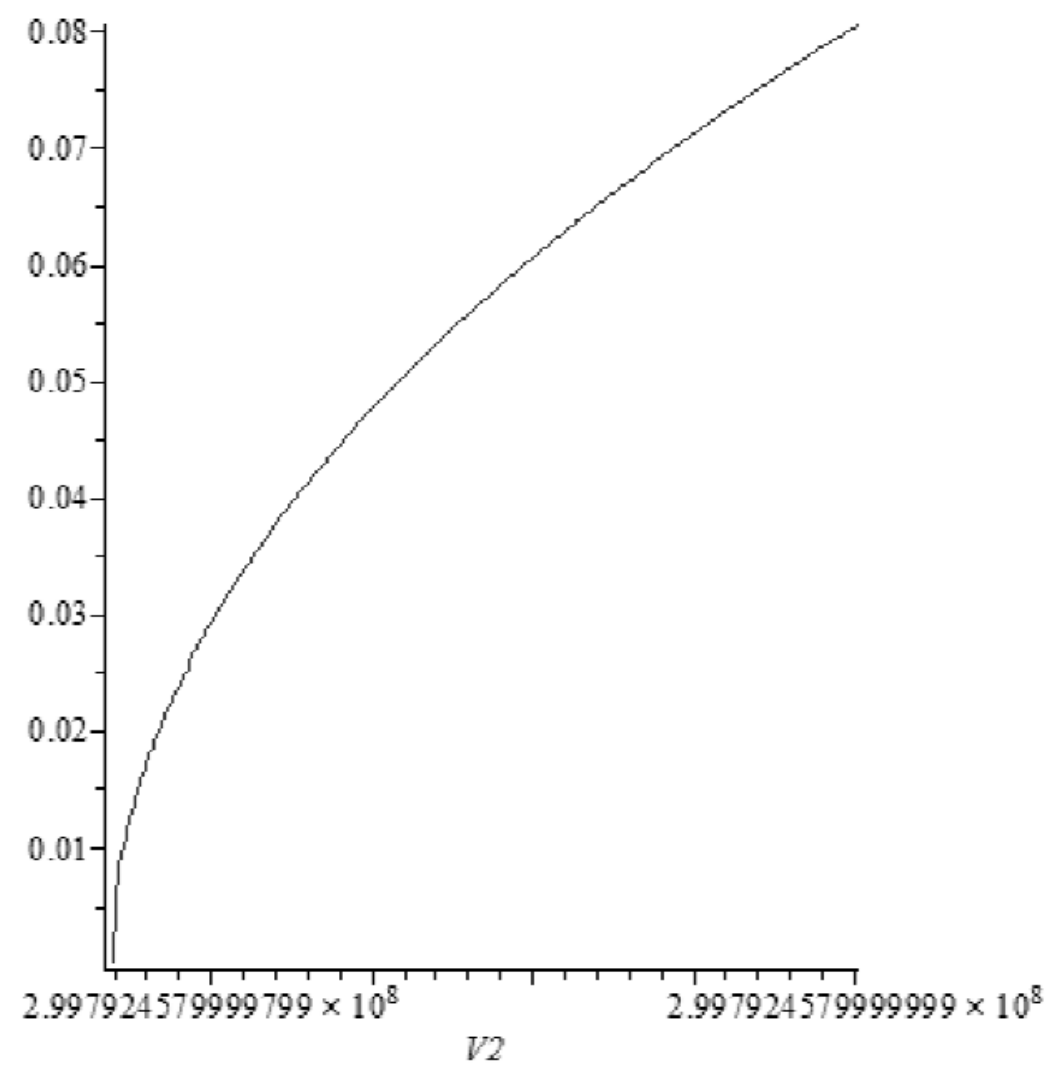

Fig. 2 Dependence of the angle $\theta$ of the photon and the "recoil" electron on the velocity of the recoil electron $\left(V_{2}\right)$. The speed of an electron is measured in meters per second. The angle of dispersion $\theta$ is measured in angular seconds.

$$
\mathrm{V} 2=-\frac{1}{v 1+\mathrm{v} 2}\left(\mathrm { c } \left(\left(4 v _ { 1 } \mathrm { h } v _ { 2 } \left(-\mathrm{h} v_{1}^{2} v_{2}+v_{1} \mathrm{~h} v_{2}^{2}+\left(\mathrm{h}_{2} v_{1}^{4} v_{2}^{2}+\right.\right.\right.\right.\right.
$$

$2 h^{2} v_{1}^{3} v_{2}^{3}+h^{2} v_{1}^{2} v_{2}^{4}+2 c^{4} m^{2} v_{1}^{2} v_{2}^{2}+c^{4} m^{2} v_{1}^{3} v_{2}+$ $\left.\left.\left.c^{4} m^{2} v_{1} v_{2}^{3}\right)^{1 / 2}\right)\right) /\left(2 c^{4} m^{2} v_{1} v_{2}+4 h^{2} v_{1}^{2} v_{2}^{2}+m^{2} c^{4} v_{1}^{2}+\right.$ $\left.\left.\mathrm{m}^{2} \mathrm{c}^{4} \mathrm{v}_{2}^{2}\right)-v_{2}+v_{1}\right)$ ),

$$
\begin{aligned}
& \mathrm{V} 2=-\frac{1}{v 1+v 2}\left(\mathrm { c } \left(-\left(4 v _ { 1 } \mathrm { h } v _ { 2 } \left(-\mathrm{h} v_{1}{ }^{2} v_{2}+v_{1} \mathrm{~h} v_{2}^{2}+\left(\mathrm{h}_{2} v_{1}^{4} v_{2}^{2}\right.\right.\right.\right.\right. \\
& +2 \mathrm{~h}^{2} v_{1}^{3} v_{2}^{3}+\mathrm{h}^{2} v_{1}^{2} v_{2}^{4}+2 \mathrm{c}^{4} \mathrm{~m}^{2} v_{1}^{2} v_{2}^{2}+\mathrm{c}^{4} \mathrm{~m}^{2} v_{1}^{3} v_{2}+ \\
& \left.\left.\left.\mathrm{c}^{4} \mathrm{~m}^{2} v_{1} v_{2}^{3}\right)^{1 / 2}\right)\right) /\left(2 \mathrm{c}^{4} \mathrm{~m}^{2} v_{1} v_{2}+4 \mathrm{~h}^{2} v_{1}^{2} v_{2}^{2}+\mathrm{m}^{2} \mathrm{c}^{4} v_{1}{ }^{2}+\right. \\
& \left.\left.\left.\mathrm{m}^{2} \mathrm{c}^{4} v_{2}^{2}\right)-v_{2}+v_{1}\right)\right), \\
& \quad V 1=\mathrm{c}\left(1-\left(\mathrm{mc}^{2} /\left(-\mathrm{h} v_{1}+\mathrm{mc}^{2} /\left(1-\mathrm{V}_{2}^{2} / \mathrm{c}^{2}\right)^{1 / 2}+\mathrm{h} v^{2}\right)\right)^{2}\right)^{1 / 2}
\end{aligned}
$$

Obviously, $\mathrm{V}_{2}$ has a real value at $\theta=0[3-5]$.

\section{References}

[1] http://www.nkj.ru/news/28446/.

[2] Kardashev N. S. Earth and the Universe. 2016. 4: 50.

[3] Perov, N. I., Eltsov, A. A. 2016. "Quasars and the Next 'Catastrophe' in Physics." In Proceedings of the All-Russian Conference on Actual Problems of Astronomy and Astronomical Education, 14-6.

[4] Perov, N. I., Eltsov, A. A. 2017. "On the Solution of the Quasar's Catastrophe Problem." Presented at the conference HEPRO VI. ISR, Moscow, Russia.

[5] Perov, N. I., Eltsov, A. A. 2017. "On the Interpretation of the 'Catastrophe' Quasars." In Proceedings of the All-Russian Astronomical Conference on Astronomy: Cognition without Borders, 203. 